\title{
Playing your heart out
}

\author{
K. D. O'Halloran ${ }^{1}$
}

Received: 17 September 2015/Accepted: 18 September 2015/Published online: 10 October 2015

(C) Royal Academy of Medicine in Ireland 2015

The twinning of heart and breath in physiology is a common theme. The regulation of cardiorespiratory coupling across a broad range of physiological demands is a function of the brain. The rhythmicity and pattern of breathing is determined by nerve cell networks in the brain, in automatic fashion. Nerve projections to muscles of the chest bring about the mechanical act of breathing. Similarly, nerve projections from the brain strongly influence the intrinsic pacemaker of the heart. The marriage of heart and breath is revealed in the brain too, with coupling in the activity of nerve cell networks influencing the expression of breath and beat. And all of this, most often, achieved without a moment's notice.

I have long thought of these paired expressions as twinned melodies orchestrated by the brain. The contrasting, yet complementary rhythms provide a physiological counterpoint, which in health culminates in a calm, cardiorespiratory tune. One can consider then, in contrast, that illness is often heard by the physician, as the discordant clamour of heart and breath: a syncopated cacophony, the din of disease, as melody turns to malady.

University College Cork hosted a symposium Playing Your Heart Out, exploring the interplay of science and music, in the Aula Maxima on 18 September 2015, as part of the Sounds from a Safe Harbour festival, which ran 17-20 September 2015 predominantly in Cork City (http:// www.soundsfromasafeharbour.com). The centrepiece of the UCC event was the contemporary classical composition Music for Heart and Breath (by Richard Reed Parry of

K. D. O'Halloran

k.ohalloran@ucc.ie

1 Department of Physiology, University College Cork, Cork, Ireland
Arcade Fire). In an unusual twist, the score is played by musicians who use their own heartbeat and breath cycle to dictate the pace and pulse of the music. Stethoscopes are employed to enable musicians to inspect and reveal their internal rhythms providing personal signatures that tumble melodically through the carefully crafted composition as a central component of the performance.

Reed Parry's concept of Music for Heart and Breath is intriguing on so many levels. The literal, real time, introspection of sorts, provides the rhythmical substrate for the elaboration, through his composition, of an intimate, soothing, near hypnotic expression. It is mesmerising. Its universality is the concept that each of us expresses individual melodies; each of us musical, entwined in a global symphony. Music for Heart and Breath is both from and for heart and breath. The emotional impact of the composition as it plays out is in turn reflected in the heart and breath of listeners, and in the musicians themselves. This organic feedback loop weaves further complexity to the performance.

The brain regions responsible for the expression of physiological heart and breath are under conscious and emotional control. It has long been recognised that emotions affect cardiorespiratory control-indeed, the response of heart and breath to affective states is part and parcel of the integrated whole body response to emotional challenges. This is recognised in our language with many expressions, such as when we note that our heart has 'skipped a beat' or when we encourage others to 'take a deep breath' or 'catch your breath', and so on. Indeed, it is interesting to note that in the English language, the word heart can be found in breath, further emphasising-for those fond of anagrams at least-the intimacy of these two bedfellows. Music is well recognised for its capacity to affect the emotional state. It is increasingly recognised that 
this partly relates to sub-conscious effects, and processing of sensory information by brain regions that constitute the body's arousal and defence systems, archaic regulatory systems that are often employed in the body's classical fight or flight response, that employs the cardiorespiratory (and other) systems as part of an integrated response to stressors. Whilst music can quickly be recognised as posing no obvious threat to the body, it nevertheless evokes a visceral response consistent with the activation of emotional centres of the brain.

Why does music evoke such a powerful emotional response? In view of the intrinsic biological rhythms that serve to maintain whole body health, ensuring homeostasis, it is tempting to consider that musical melody moves us, owing to a harmonic interplay with our own rhythms, tapping into the emotional centres of the brain and thereafter awakening the sites that serve to process this altered state, which often struggles to pinpoint with accuracy why we feel such a connection to certain compositions. Indeed, for some, the experience in real time, appears to hijack the capacity to articulate the feeling through breath at all, leaving them, quite literally, speechless. Similar processing of visual art, literature and poetry (and of course a host of other joys to behold) can achieve similar outcomes, but there is something quite special and encompassing about music.

Professor John Cryan, Head of the Department of Anatomy and Neuroscience, UCC co-hosted the symposium providing expertise on sensing, processing and responding in the nervous system to the emotional impact of the music. Duet for Heart and Breath was performed by Richard Reed Parry and Nadia Sirota.

As a physiologist, and experimentalist, I am intrigued by the rich landscape that Music for Heart and Breath provides for observation and measurement. The many questions that arise serve to quicken my pulse-a career hazard. Beyond that, however, the many questions and answers that Music for Heart and Breath provides in respect of the intimate relationship between musician, composition and audience are spellbinding and a joy to ponder. 